\title{
Incorporating Basic Optical Microscopy in the Instrumental Analysis Laboratory
}

published in the Journal of Chemical Education, 2011, 88(12), 1716-1719.

Paul A. Flowers, Department of Chemistry and Physics, University of North Carolina at

Pembroke, Pembroke NC 28372-1510, United States, paul.flowers@uncp.edu

\begin{abstract}
A simple and versatile approach to incorporating basic optical microscopy in the undergraduate instrumental analysis laboratory is described. Attaching a miniature CCD spectrometer to the video port of a standard compound microscope yields a visible microspectrometer suitable for student investigations of fundamental spectrometry concepts, basic principles of optical microscopy, and some interesting phenomena unique to spectral measurements performed at the microscale. Results from several such investigations and ideas for additional applications are presented.
\end{abstract}

\section{KEYWORDS}

Upper-Division Undergraduate, Laboratory Instruction, Analytical Chemistry, Hands-On Learning / Manipulatives, Calibration, Instrumental Methods, UV-Vis Spectroscopy 
A considerable fraction of industrial positions for bachelor degree chemists include chemical analysis as a primary job function. For example, data collected by the American Chemical Society indicate that roughly one-half of nonacademic/nonmanufacturing industrial chemists are employed in "analytical/research service" positions (1). Of the chemists employed in manufacturing positions, it's likely that many are engaged in chemical analysis as a significant job responsibility (quality assurance/quality control, product testing, etc.). Though these data represent chemists of all degree levels, it is arguably common knowledge that the majority of such bench-analyst jobs are held by bachelor degree chemists. Accordingly, undergraduate chemistry programs should assess the content of analytical and instrumental analysis courses and correlate the knowledge and skills provided by these courses to the expectations of chemical industry (2-5).

Recent surveys have shown several disparities between the analytical techniques commonly utilized by chemists in industry and those encountered in typical undergraduate chemistry programs $(6,7)$. This disparity is particularly distinct for optical microscopy, a technique routinely used in many industrial settings (8) but rarely encountered in the undergraduate chemistry laboratory. Results presented in reference 6 showed that more than one-half (55\%) of industry respondents cited optical microscopy as a technique with which new chemistry hires should have practical experience. According to a study by the same authors (7), only $3 \%$ of respondents from academia reported including optical microscopy in their laboratory curricula.

The seemingly discordant absence of optical microscopy from the undergraduate chemistry lab is not at all a new issue, being first addressed in this Journal more than eighty 
years ago (9). A handful of experiments featuring the standard optical microscope have been published in the pedagogical literature since then, most involving microscopic observations of physical and chemical processes, i.e., "chemical microscopy" (10-15). In addition, several publications have detailed the construction, modification and/or application of optical microscopes for variable-temperature measurements (16-21), interferometric measurement of diffusion in liquids (22), and single-molecule fluorescence (23-25). To the best of this author's knowledge, none of the commercially available laboratory manuals for analytical chemistry or instrumental analysis courses contain experiments featuring optical microscopy.

Rather than add a new lab project to an already full curriculum, or replace an existing project to address this disparity, a conventional spectrophotometry project for undergraduate instrumental analysis has been revised to incorporate the use of optical microscopy. Attaching a

miniature CCD spectrometer to the video port of a typical compound microscope yields a visible microspectrophotometer suitable for student investigation of fundamental spectrometry concepts, basic concepts of optical microscopy, and some interesting phenomena unique to spectral measurements performed at the microscale. This paper describes several such investigations, including examples of typical student data and ideas for additional applications.

\section{EXPERIMENTAL}

\section{Instrumentation}

A visible microspectrophotometer was assembled by connecting the video port of a compound microscope (Micromaster II, Fisher Scientific, Pittsburgh PA, USA) to the entrance slit of a miniature CCD spectrometer via a single fiber optic patch cable and an SMA-to-Cmount adapter (model USB 2000, P600-025-VIS-NIR and C-MOUNT-MIC, respectively, Ocean 
Optics, Inc., Dundee FL, USA). A digital microscopy camera (Infinity-2, Lumenera Corporation, Ottawa ON, Canada) was used in some cases to obtain photomicrographs of the samples under study, though its use is entirely optional. The SpectraSuite software platform (Ocean Optics) operating in a Windows XP environment was used for spectrometer control and data acquisition and processing. An illustration of the experimental setup is shown in Figure 1.

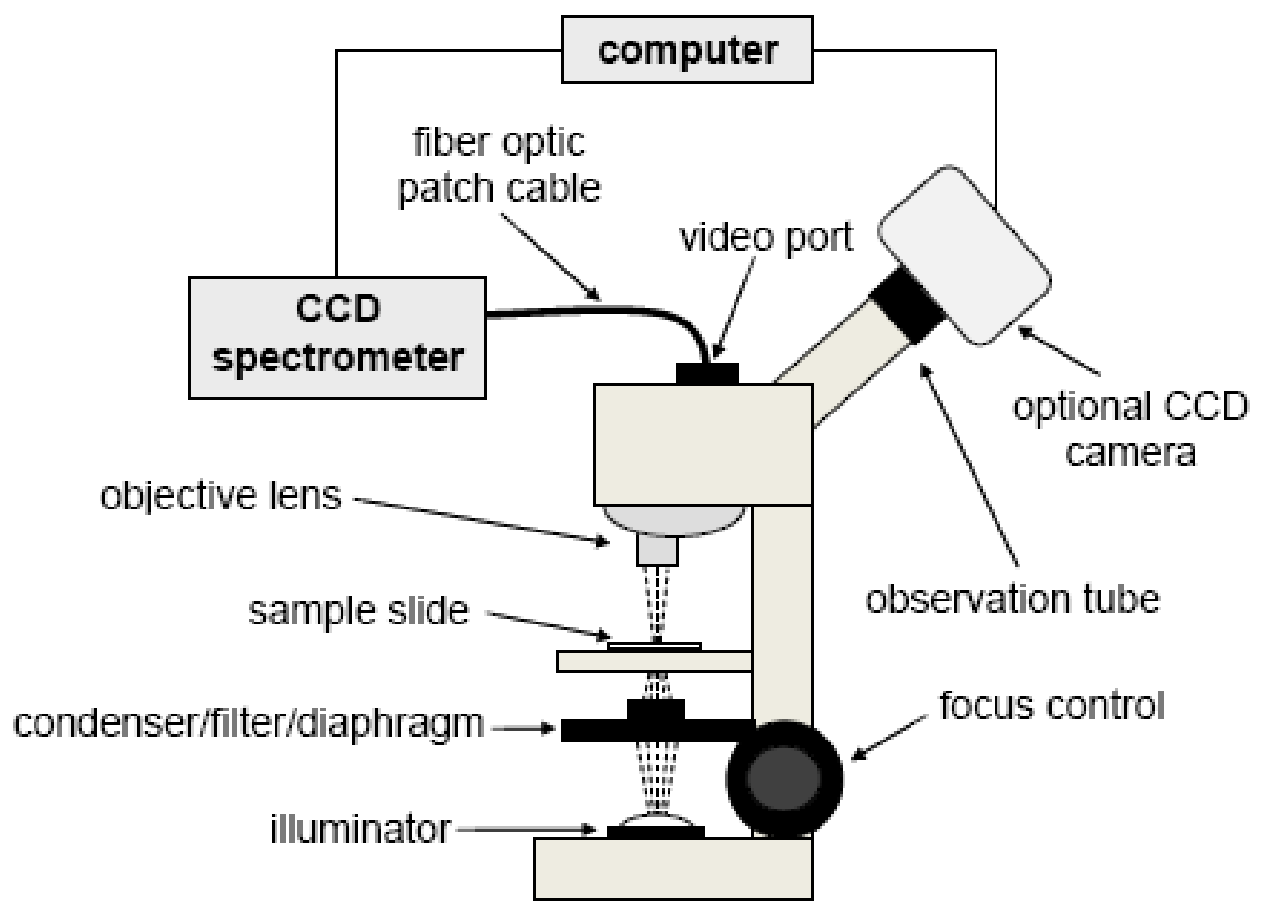

Figure 1. Schematic of the microspectrophotometer.

\section{Procedures}

Students were provided instructions for performing "instrument orientation" work in a three-hour laboratory session that demonstrated signal enhancement schemes (ensemble averaging, integration, smoothing) and photometric principles (wavelength/color correlation, stray light, Beer's law) along with basic concepts in optical microscopy (sample stage and focus 
adjustments, trans-illumination, magnification). A typical set of student instructions for this lab work is provided in the supplemental information accompanying this article.

The lab-intensive format of our instrumental analysis course includes additional lab meetings following the orientation sessions during which students conduct more in-depth work with selected instruments. Students who choose the microspectrometer for this additional work investigate more advanced aspects, for example: mapping the effective aperture of the fiber optic patch cable; configuring the microscope to permit reflectance measurements on opaque samples via epi-illumination; measuring absorbance spectra of individual emulsion droplets; etc. Detailed written instructions are not provided for such in-depth work, rather students are required to consult the professor for guidance in developing appropriate experimental procedures.

\section{RESULTS AND DISCUSSION}

\section{Stray Light}

Ambient light from the laboratory's overhead fluorescent lamps can enter the microspectrometer via both the objective and eyepiece lenses. With the microscope illuminator powered down, students investigate this source of stray light by measuring spectra under varying microscope configurations. With the microscope's sample stage empty and its eyepiece capped, stray light is minimal, and the measured spectrum exhibits several very weak, narrow-band features characteristic of mercury vapor-based fluorescent lamps (26). These spectral features become significantly more intense when a piece of white filter paper is placed on the sample stage, as it serves to diffusely reflect light into the microscope's objective. Many students are initially puzzled by the further increase in this stray light signal observed when the eyepiece is uncapped, and the fact that this signal remains considerable even when the filter paper is 
removed from the sample stage. With a bit of thought, and perhaps guidance from the professor, they soon realize this behavior is due to the beam splitter in the microscope head, which permits simultaneous passage of light from the objective to both the video port and eyepiece (27).

\section{Magnification}

Measuring emission spectra for the microscope's illuminator using different magnifications provides students an instrumental verification of the generalization that images appear darker as magnification is increased. With an empty slide on the sample stage, spectra of the illuminator operated at a constant power are measured using the $4 \mathrm{X}, 10 \mathrm{X}, 40 \mathrm{X}$ and $100 \mathrm{X}$ objectives. Typical spectra measured in this fashion, along with a plot of peak signal versus magnification factor, are shown in Figure 2. Since signal is proportional to the cross-sectional area of the optical beam collected by the microscope's objective, and this cross-sectional area is typically inversely proportional to the square of the lateral or transverse magnification, an inverse-square dependence of signal on magnification is expected (28). This relationship is supported by the data in Figure 2, which is nicely fitted to an inverse-square regression equation $\left(\mathrm{R}^{2}=0.99\right.$, exponent=-1.9). Students are challenged in a post-lab quiz item (see supplemental information) to confirm that the signal-to-noise ratio is inversely proportional to magnification. These measurements are also helpful in leading students to consider some practical aspects of microspectrometry, namely, the trade-off between spatial resolution and spectral quality. 

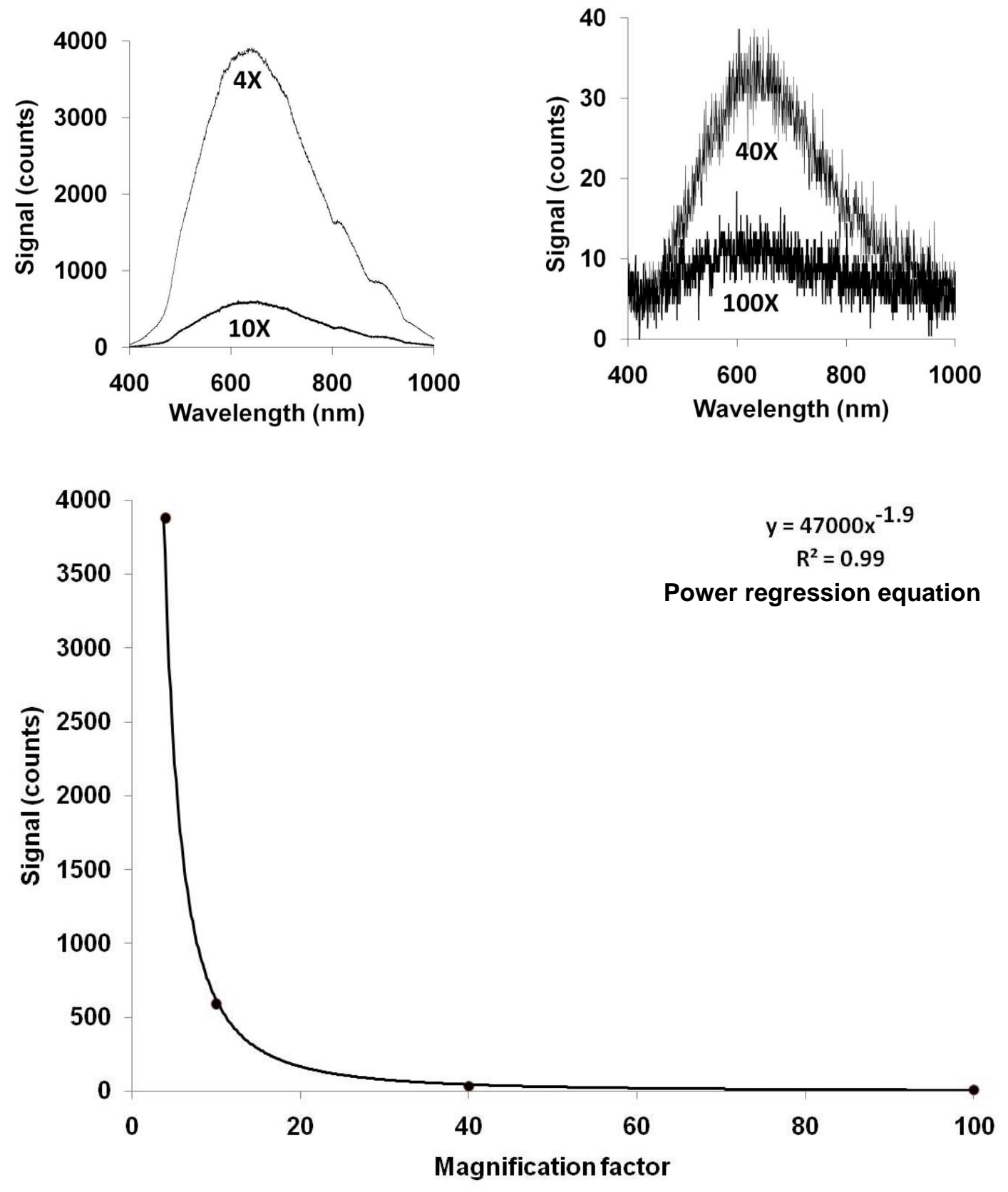

Figure 2. Spectra of the microscope's illuminator measured using different objectives (upper) and plot of peak signal versus magnification factor (lower). Inset equation obtained via power regression on the plotted data. 


\section{Transillumination}

Students are provided a microscope slide containing several "targets" consisting of $\sim 1$ $\mathrm{mm}^{2}$ pieces of transparent colored plastic cut from the label tabs of binder separator sheets and secured to the surface of the slide with transparent adhesive tape. A colorless target is used to acquire a reference spectrum against which absorbance spectra for the colored targets are measured (see Figure 3). The real-time spectral display of the data acquisition software is very useful for these measurements, as it permits students to fine-tune the sample position (XY) and focus $(Z)$ to yield the highest quality spectra. Regarding the former parameter, students learn that the optimal sample position for spectral observation is not the center of the field-of-view, an unintended feature of the microspectrometer's optical train resulting from inexact positioning of the spectrometer's input fiber on the microscope head (our microscope's C-mount connector was missing components required for a precise connection). This off-center alignment may be investigated through subsequent in-depth work as described in the "Effective Spectral Aperture" section below.

\section{Path Length}

As optional work during either the instrument orientation lab meeting (if time permits) or the subsequent in-depth session, students investigate photometric aspects of the microspectrometer by performing a typical calibration using food dye solutions. Instead of using a cell to contain the samples, however, measured volumes of the standards are pipetted directly onto the surface of a glass microscope slide. The aqueous sample drops efficiently wet the surface of bare slides, spreading to yield an irreproducibly thin layer of solution and, consequently, calibration curves of relatively low sensitivity, poor precision, and no obvious 


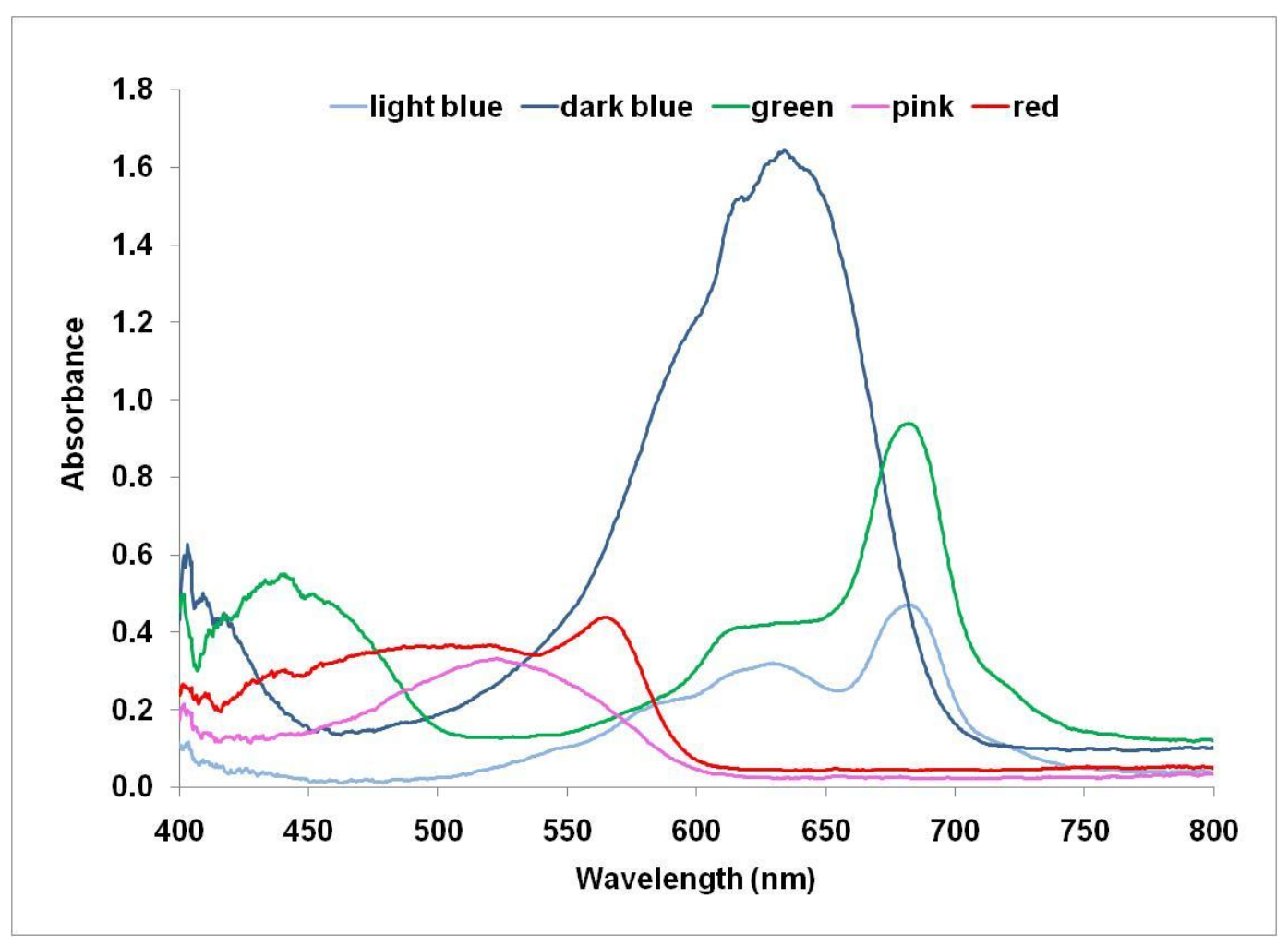

Figure 3. Absorbance spectra of $\sim 1 \mathrm{~mm}^{2}$ colored plastic targets measured with the microspectrometer (10X objective, $0.1 \mathrm{~s}$ integration time, 1 scan collected, 12-pt smooth).

dependence on the employed sample volume. Covering the slide with household plastic wrap provides a hydrophobic surface that permits the sample drops to bead nicely, increasing the effective path length (thus sensitivity) and the precision of the absorbance measurements. Assuming the sample drops are approximately hemispherical, a cube-root dependence of path length is expected, and has been observed as shown by the student data presented in Figure 4. 

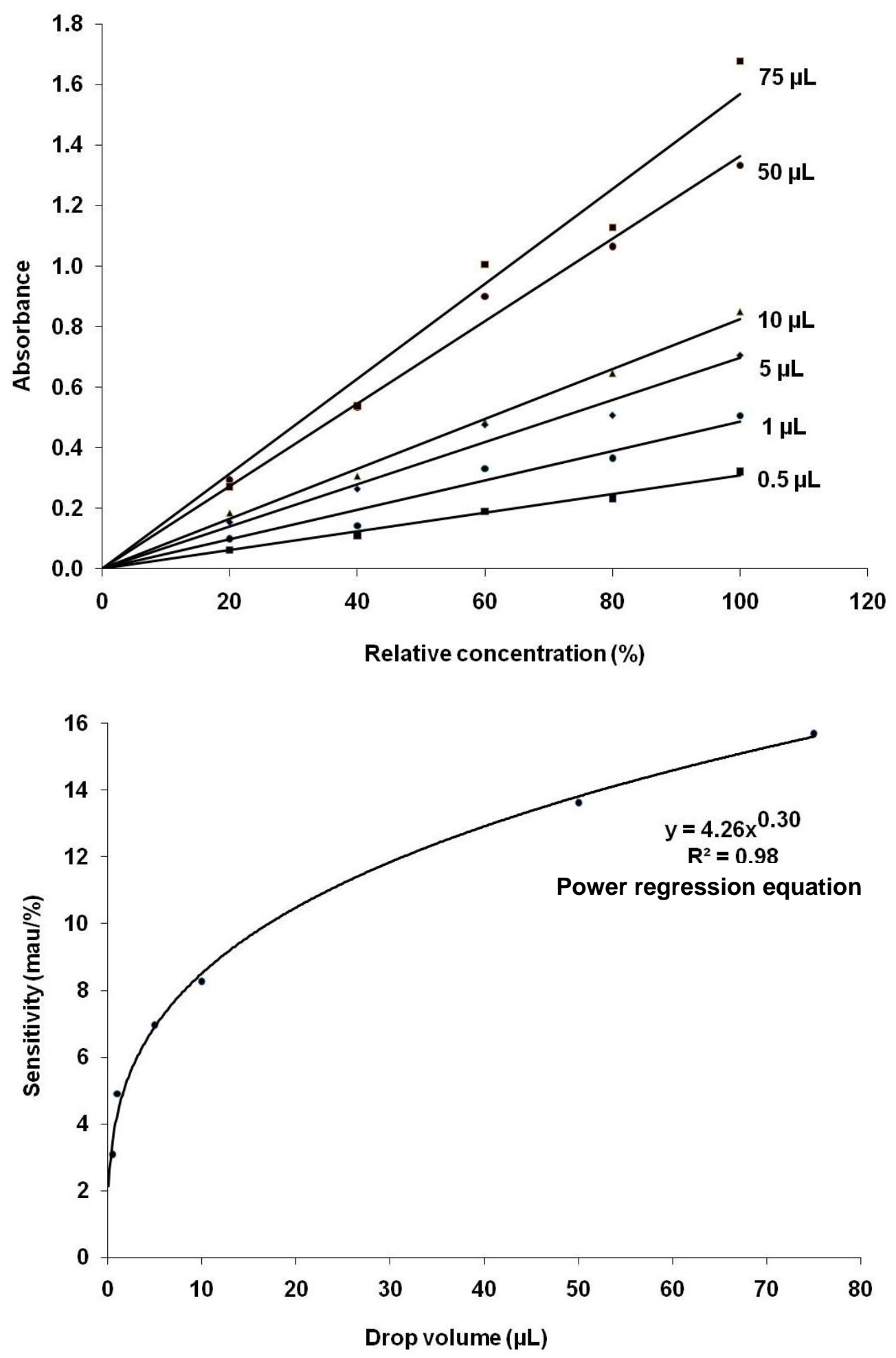

Figure 4. Calibration curves for green food coloring standards at various sample drop volumes (upper) and plot of sensitivity (calibration curve slope) versus sample drop size. Absorbance measured at $630 \mathrm{~nm}$ and a nominal bandwidth of $2 \mathrm{~nm}$. Inset equation obtained via power regression on the plotted data. 


\section{Effective Spectral Aperture}

As mentioned above in the "Transillumination" section, the optical train of the microspectrometer, as assembled, entails a slightly off-center placement of the fiber optic patch cable relative to the visual field-of-view. The effective aperture of the patch cable is also considerably smaller than the total field-of-view area, and so it is interesting and useful to have students map the source intensity during an in-depth lab session. Briefly, the procedure requires the use of a grid eyepiece reticule (calibrated with a stage micrometer, if absolute position values are desired) and a pinhole aperture (100 $\mu \mathrm{m}$ diameter or lesser) mounted on the sample stage. Source intensities are measured with the pinhole positioned within the reticule squares, and these intensity values are plotted versus the pinhole's XY-position within the focal plane as shown in Figure 5.

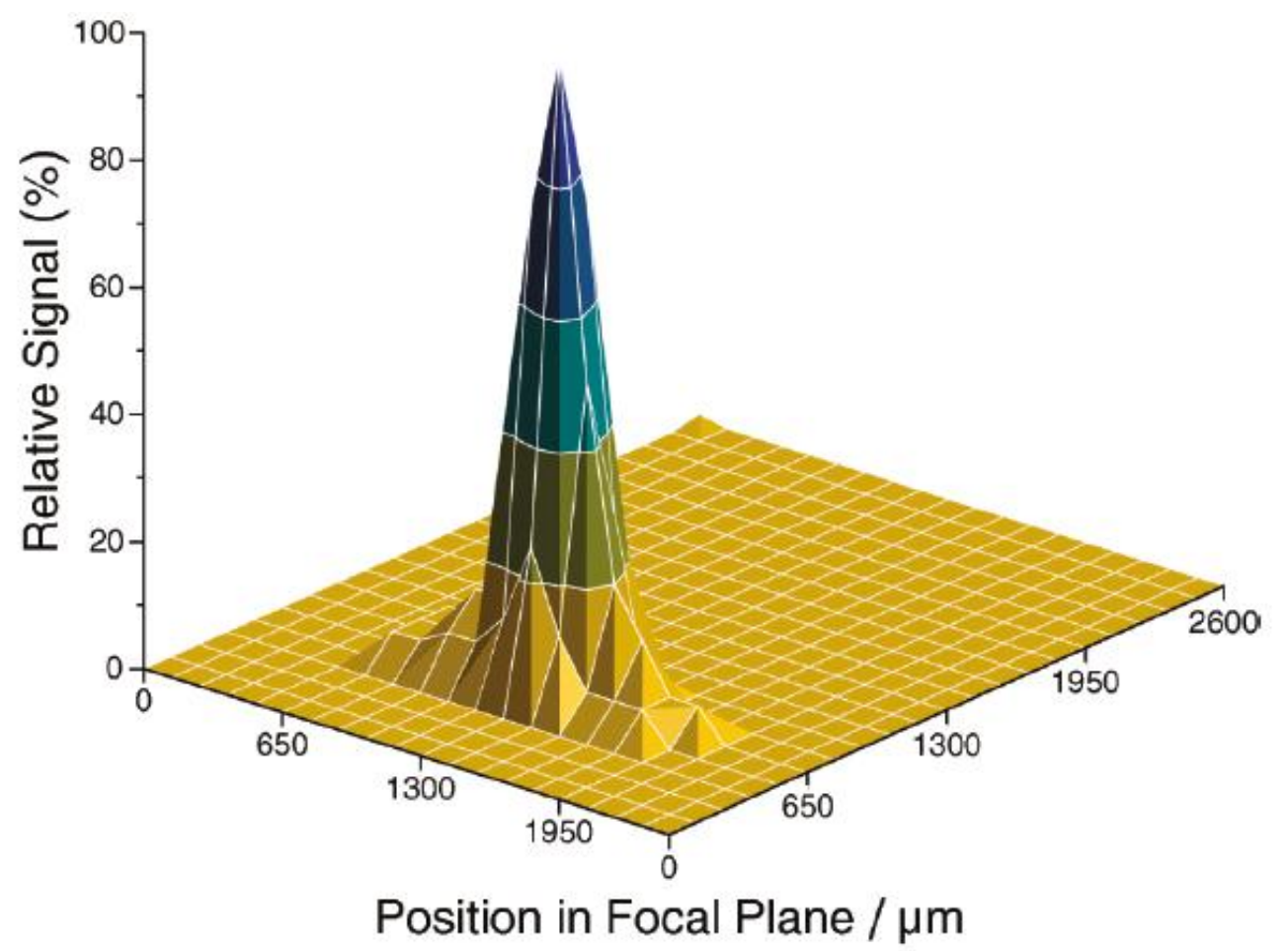

Figure 5. Source intensity map showing the effective aperture of the microspectrometer's fiber optic patch cable (4X objective). 


\section{CONCLUSIONS}

The laboratory work described in this paper represents a practical means of providing students experience with basic optical microscopy techniques that are widely used in industry but largely absent from the undergraduate chemistry curriculum. By integrating microscopy into a traditional spectrometry lab project, additional content is added without the need to sacrifice existing material. In addition to the lab investigations reported here, the microspectrometer is suitable for demonstration of numerous other spectrometry and microscopy concepts. The equipment requirements are modest, and the spectrometer's optical configuration may be easily altered to permit other applications. For example, an external light source may be introduced to the microscope's optical train via its eyepiece to yield an epi-illumination arrangement suitable for reflectance and fluorescence measurements. Finally, a wide variety of chemical systems are amenable to microspectrometric analysis (forensic, biological, materials science, etc.), providing instructors flexibility in the selection of topics likely to be most engaging for their students.

\section{HAZARDS}

As for any optical microscope, students should be cautioned to minimize the risk of eye strain and potential retinal damage by viewing samples through the eyepiece for brief time intervals with both eyes open, and by employing the lowest practical illuminator intensity. The optional use of a video camera (see "Experimental" section) is recommended eliminate these risks. 


\section{ACKNOWLEDGMENT}

The author gratefully acknowledges the students of his spring 2009 and 2010

Instrumental Analysis courses and undergraduate research assistants Josie Torrence and Cilia Iluku for conducting the experimental work described in this article.

\section{LITERATURE CITED}

1. Heylin, M. Chem. Eng. News 2008, 9, 37-47.

2. Harwood, J.J. J. Chem. Educ. 1989, 66(11), A268-A271.

3. Harris, H.H.; O’Brien, J.J. J. Chem. Educ. 1992, 69(10), A266-A268.

4. Jones, B.T. J. Chem. Educ. 1992, 69(10), A268-A269.

5. Girard, J.E.; Diamant, C.T. J. Chem. Educ. 2000, 77(5), 646-648.

6. Fahey, A.; Tyson, J. Anal. Chem. 2006, 78, 4249-4254.

7. Fahey, A.M.; Tyson, J.F. Chem. Educator 2006, 11, 445-450.

8. For example, see the 27 "microscopy" application notes at the Keyence Corporation of America website, http://www.keyence.com/applications/index.php (accessed 28 Dec 2010).

9. Chamot, E.M.; Mason, C.W. J. Chem. Educ. 1928, 5(3), 258-268.

10. Chamot, E.M.; Mason, C.W. J. Chem. Educ. 1928, 5(1), 9-24.

11. Schaeffer, H.F. J. Chem. Educ. 1942, 19(1), 15-16.

12. Thiessen, G.W.; Beste, L.F. J. Chem. Educ. 1942, 19(7), 331-332.

13. Benedetti-Pichler, A.A.; Vikin, J. J. Chem. Educ. 1966, 43(8), 421-422.

14. Winokur, R.; Monroe, M. J. Chem. Educ. 1985, 62(2), 157. 
15. Lam-Erwin, C.-Y.; Sprague, J. J. Chem. Educ. 1992, 69(10), 855.

16. Wallace, E.H.; Willard, M.L. J. Chem. Educ., 1931, 8(4), 706-711.

17. Bonner, W.A. J. Chem. Educ. 1946, 23(12), 601-602.

18. Hutchings, L.E. J. Chem. Educ. 1951, 28(4), 214-215.

19. Glasser, L.; Miller, R.P. J. Chem. Educ. 1965, 42(2), 91-95.

20. Verbit, L.; Halbert, T.R. J. Chem. Educ. 1971, 48(11), 773-774.

21. McGhie, A.R. J. Chem. Educ. 1976, 53(10), 637.

22. Nishijima, Y.; Oster, G. J. Chem. Educ. 1961, 38(3), 114-117.

23. Zimmermann, J.; van Dorp, A.; Renn, A. J. Chem. Educ. 2004, 81(4), 553-557.

24. Protasenko, V.; Hull, K.L.; Kuno, M. Chem. Educator, 2005, 10(4), 269-282.

25. Ito, T. J. Chem. Educ. 2008, 85(5), 680-682.

26. Goode, S.R.; Metz, L.A. J. Chem. Educ. 2003, 80(12), 1455-1459.

27. Davidson, M.W., "Optical Microscopy Primer: Optical Microscope Components”, http://micro.magnet.fsu.edu/primer/anatomy/componenthome.html (accessed 4 Jun 2011).

28. Davidson, M.W., “Optical Microscopy Primer: Image Brightness”, http://micro.magnet.fsu.edu/primer/anatomy/imagebrightness.html (accessed 4 Jun 2011).

\section{SUPPORTING INFORMATION AVAILABLE}

Instructor notes; student instructions; post lab quiz. This material is available via the Internet at http://pubs.acs.org. 